\title{
Polysomnographic phenotypes and their cardiovascular implications in obstructive sleep apnoea
}

\author{
Andrey V Zinchuk, ${ }^{1}$ Sangchoon Jeon, ${ }^{2}$ Brian B Koo, ${ }^{3}$ Xiting Yan, ${ }^{1}$ Dawn M Bravata, ${ }^{4}$ \\ Li Qin, ${ }^{5}$ Bernardo J Selim, ${ }^{6}$ Kingman P Strohl, ${ }^{7}$ Nancy S Redeker, ${ }^{2}$ John Concato, ${ }^{1,8}$ \\ Henry K Yaggi ${ }^{1}$
}

- Additional material is published online only. To view please visit the journal online (http://dx.doi.org/10.1136/ thoraxjnl-2017-210431).

For numbered affiliations see end of article.

\section{Correspondence to}

Dr Henry K Yaggi, Department of Medicine, Yale University School of Medicine, 300 Cedar Street, New Haven, CT 06443, USA; henry.yaggi@yale.edu

Part of this work was presented at as a poster discussion presentation: Zinchuk AV, Jeon S, Koo BB, et al. Clinically Relevant Phenotypes of Obstructive Sleep Apnea. Am J Respir Crit Care Med 2016;193:A6380.

Received 25 April 2017 Revised 13 July 2017 Accepted 14 August 2017

Published Online First 21 September 2017

\section{SLinked}

- http://dx.doi.org/10.1136/ thoraxjnl-2017-210943

Check for updates

To cite: Zinchuk $A V$, Jeon $S$, Koo BB, et al. Thorax

2018:73:472-480.

\section{ABSTRACT}

Background Obstructive sleep apnoea (OSA) is a heterogeneous disorder, and improved understanding of physiologic phenotypes and their clinical implications is needed. We aimed to determine whether routine polysomnographic data can be used to identify OSA phenotypes (clusters) and to assess the associations between the phenotypes and cardiovascular outcomes. Methods Cross-sectional and longitudinal analyses of a multisite, observational US Veteran $(n=1247)$ cohort were performed. Principal components-based clustering was used to identify polysomnographic features in OSA's four pathophysiological domains (sleep architecture disturbance, autonomic dysregulation, breathing disturbance and hypoxia). Using these features, OSA phenotypes were identified by cluster analysis (K-means). Cox survival analysis was used to evaluate longitudinal relationships between clusters and the combined outcome of incident transient ischaemic attack, stroke, acute coronary syndrome or death.

Results Seven patient clusters were identified based on distinguishing polysomnographic features: 'mild', 'periodic limb movements of sleep (PLMS)', 'NREM and arousal', 'REM and hypoxia', 'hypopnoea and hypoxia', 'arousal and poor sleep' and 'combined severe'. In adjusted analyses, the risk (compared with 'mild') of the combined outcome (HR $(95 \% \mathrm{Cl}))$ was significantly increased for 'PLMS', (2.02 (1.32 to 3.08)), 'hypopnoea and hypoxia' (1.74 (1.02 to 2.99)) and 'combined severe' (1.69 (1.09 to 2.62)). Conventional apnoeahypopnoea index (AHI) severity categories of moderate $(15 \leq \mathrm{AHI}<30)$ and severe $(\mathrm{AHI} \geq 30)$, compared with $\mathrm{mild} /$ none category $(\mathrm{AHI}<15)$, were not associated with increased risk.

Conclusions Among patients referred for OSA evaluation, routine polysomnographic data can identify physiological phenotypes that capture risk of adverse cardiovascular outcomes otherwise missed by conventional OSA severity classification.

\section{INTRODUCTION}

Obstructive sleep apnoea (OSA) is increasingly recognised as a complex and heterogeneous syndrome. ${ }^{1}$ Categorising such conditions into smaller, more homogeneous categories ('phenotypes') can help advance understanding of pathophysiology, develop targeted treatments and

\section{Key messages}

What is the key question?

- Can routine polysomnographic data and unsupervised learning methods be used to identify subgroups ('phenotypes') of obstructive sleep apnoea (OSA) patients, and are those subgroups associated with increased risk of adverse cardiovascular outcomes or death?

What is the bottom line?

- In patients referred for OSA evaluation, we identified physiological phenotypes that captured risk of adverse cardiovascular outcomes otherwise missed by conventional OSA severity classification using the apnoeahypopnoea index.

Why read on?

- We show that physiologically distinct groups of patients (eg, with higher prevalence of periodic limb movements of sleep, arousals, hypoxia and so on) exist within each of the traditional OSA severity categories and that these groups may have implications for treatment and risk of cardiovascular events or death.

improve both prognostication and risk stratification for clinical trials. Polysomnography enables collection of multiple types of physiological data, which results in numerous metrics by which patients with OSA can be characterised. Previously suggested OSA phenotypes were based primarily on occurrence of respiratory events during specific sleep states, ${ }^{2}{ }^{3}$ body position ${ }^{4}$ or hypoxia. ${ }^{5}$ These prior studies used approaches that identified the phenotypes a priori, based on a single or few characteristics, and therefore could have missed more complex and (up to this point) unidentified OSA subgroups. ${ }^{6}$

The clinical relevance, including prognostic implications, of such polysomnogaphic phenotypes remains largely uncertain. OSA is a well-known independent risk factor for cardiovascular disease (CVD) and death. ${ }^{7-9}$ Much of the epidemiological work linking OSA and CVD outcomes, however, is focused primarily on the apnoea-hypopnoea index (AHI) ${ }^{10}$ which represents a small fraction of the physiological data generated by polysomnography. 
Recent studies suggest that the AHI alone may not fully capture the physiological processes (eg, hypoxaemic burden ${ }^{11}$ sleep fragmentation ${ }^{12}$ or periodic limb movements of sleep (PLMS) $)^{13}$ implicated in development of adverse cardiovascular outcomes.

Cluster analysis, a type of unsupervised learning methodology, can integrate multiple characteristics (such as polysomnographic metrics) without a priori groupings to identify unique patient categories. ${ }^{14}$ In other heterogeneous disorders such as asthma, COPD and heart failure, phenotypes with different prognoses and treatment responsiveness were identified using cluster analysis. ${ }^{15-17}$ In OSA, research using unsupervised learning methods to identify patient subgroups based on polysomnographic metrics is scant. ${ }^{18}$ Associations of such subgroups with cardiovascular outcomes remain largely unexplored.

Our objective was to use routine polysomnographic data to capture OSA's physiological heterogeneity in relation to clinically relevant cardiovascular outcomes. Specifically, we hypothesised that unique clusters (phenotypes) of patients could be identified by applying unsupervised learning methods to polysomnographic data and that the clusters would be differentially associated with risk of adverse cardiovascular outcomes (acute coronary syndrome (ACS), transient ischaemic attack (TIA), stroke or death.

\section{METHODS}

A comprehensive description of methodological details is provided in the online supplementary file.

\section{Participants and measures}

The Determining Risk of Vascular Events by Apnea Monitoring (DREAM) study consists of 2041 veterans from three Veterans Affairs Centers (West Haven, Connecticut; Cleveland, Ohio; and Indianapolis, Indiana) that underwent OSA evaluation between 2000 and 2004, with follow-up through $2012^{20}$. At enrolment (ie, time of polysomnography), data were collected on demographic and anthropometric characteristics, cardiovascular risk factors, medical comorbidities and other measures, as detailed previously. ${ }^{20}$ Cardiovascular risk was ascertained using the Framingham risk score (FRS; including age, sex, smoking status, presence of diabetes, antihypertensive medication, systolic blood pressure, total cholesterol and high-density lipoprotein levels ${ }^{21}$ and comorbidity burden by the Charlson comorbidity index (CCI). ${ }^{22}$ Polysomnographic scoring, variable definitions, classification and continuous positive airway pressure (CPAP) treatment adherence (regular vs not regular) are described in the online supplementary file. All polysomnographic scoring was performed at a single centre (West Haven, Connecticut). The primary outcome was the composite of incident ACS, TIA, stroke or death from any cause. Rationale for composite outcome selection and its adjudication are described previously ${ }^{20}$ and in the online supplementary file.

\section{Polysomnographic variable selection and analytic sample}

Sixty-five polysomnographic variables were sorted into four domains, representing the pathophysiological processes previously reported to be in the causal pathway between OSA and CVD: breathing disturbance, autonomic dysregulation, hypoxaemia and sleep disturbance. ${ }^{1023}$ Redundant variables (eg, time in bed) were excluded if other included variables reflected similar physiological parameters (eg, total sleep time and efficiency). Categorical variables (eg, snoring), and variables with $>5 \%$ missing data, were excluded due to cluster analysis requirements (see online supplementary file for details).
Twenty-nine polysomnographic variables (see online supplementary table E1) were ultimately selected for variable reduction analysis.

From the source population $(n=2041)$, the final analytic sample $(n=1247)$ was obtained. Patients with missing data on any polysomnographic variables $(n=482)$ were excluded due to cluster analysis requirements. Patients with inconsistently abstracted polysomnograms or scoring discrepancies $(n=308)$, or those with $<15 \mathrm{~min}$ of sleep $(\mathrm{n}=4)$ were also excluded.

\section{Statistical analyses}

A two-step variable reduction analysis was used to identify unique polysomnographic features within each domain. First, principal components-based clustering was used to identify groups of variables highly correlated with their own cluster and uncorrelated with others. ${ }^{24}$ Second, polysomnographic features were selected to retain $>75 \%$ of total variance within each domain, using the least number of features that were judged to be clinically interpretable (see online supplementary figure E1 and table E2).

We employed K-means analysis to generate clusters, based on polysomnographic feature scores. Silhouette widths (a cluster quality measure $)^{25}$ and clinical interpretability were used to select the number of clusters (detailed description in online supplementary file). Jaccard coefficient of bootstrap cluster samples was used to evaluate cluster stability. ${ }^{26}$

We assessed cross-sectional contrasts between clusters, using analysis of variance or $\mathrm{X}^{2}$ tests. The relationship between OSA clusters and primary outcome was evaluated with unadjusted Cox proportional hazards models and with models adjusting for FRS and CPAP use. The proportional hazards assumption for the model comparing each cluster to the 'mild' group was assessed using the cumulative hazard function: $H(t)=-\log S(t)$. Sensitivity analyses incorporated the clusters and CPAP use with (1) CCI and (2) home oxygen use at baseline (all sensitivity analyses are reported in the online supplementary file). Analyses were also performed with CVD-specific mortality as part of the composite outcome. We repeated the analysis using AHI severity categories (mild/none: $\mathrm{AHI}<15$; moderate: $15 \leq \mathrm{AHI}<30$; and severe: $\mathrm{AHI} \geq 30$ ), in place of cluster membership, to assess whether risk was captured by conventional OSA severity categories. To evaluate for differences between the analytic sample and excluded patients, we compared the baseline risk (FRS and $\mathrm{CCI}$ ) and primary outcomes between these groups (online supplementary file). SAS V.9.3 was used for all analyses. Institutional review board at each centre approved the study (WHVA HIC \#0001).

\section{RESULTS}

The study population was predominantly male, with an average age of $58.3 \pm 11.7$ years, mean AHI of $25.0 \pm 29.7$ events/hour and 29\% prevalence of CVD (table 1). The 29 polysomnographic variables were reduced to 17 features that preserved $83 \%$ of total variance (see online supplementary table E3) and were judged to be clinically interpretable. Patient-level cluster analysis resulted in seven OSA subgroups (clusters A - G) supplementary clusters A-G), labeled based on the most distinguishing features (table 2). Feature distributions are shown in online supplementary figure E1 and table E6. Given that OSA severity is conventionally expressed according to AHI-based categories (none/mild, moderate and severe) and to highlight heterogeneity within each category, cluster descriptions below are grouped by conventional OSA severity. 
Table 1 Summary of patient characteristics in the analytic sample $(n=1247)$ at baseline

\begin{tabular}{|c|c|}
\hline Variable & $\begin{array}{l}\text { Mean } \pm S D \\
\text { Median }(I Q R)^{*} \text { or } N(\%)\end{array}$ \\
\hline Age (years) & $58.3 \pm 11.7$ \\
\hline Gender (men) & $1183(94.9)$ \\
\hline Ethnicity (white) & $978(80.0)$ \\
\hline Employed & $425(34.8)$ \\
\hline BMI $\left(\mathrm{kg} / \mathrm{m}^{2}\right)$ & $34.6 \pm 7.3$ \\
\hline ESST & $10.8 \pm 5.8$ \\
\hline AHI (events/hour)* & $12.0(33.6)$ \\
\hline AHI (events/hour) & $25.0 \pm 29.7$ \\
\hline None/mild OSA $(\mathrm{AHI}<15)$ & $676(54.2)$ \\
\hline Moderate OSA $(15 \leq \mathrm{AHI}<30)$ & $198(15.9)$ \\
\hline Severe OSA $(A H I \geq 30)$ & $373(29.9)$ \\
\hline CPAP use $\ddagger$ & $402(32.9)$ \\
\hline Current smoking & $427(36.0)$ \\
\hline Current alcohol use & $581(49.8)$ \\
\hline Current drug use & $67(5.9)$ \\
\hline Home oxygen use & $87(7.2)$ \\
\hline Hypertension & $868(71.0)$ \\
\hline Diabetes & $382(31.2)$ \\
\hline Coronary artery disease§ & $238(19.5)$ \\
\hline Congestive heart failure & $137(11.2)$ \\
\hline Stroke or TIA & $100(8.2)$ \\
\hline Prior CVDף & $359(29.0)$ \\
\hline Atrial fibrillation & $98(8.0)$ \\
\hline Renal failure & $62(5.1)$ \\
\hline Chronic lung disease & $396(32.5)$ \\
\hline Cancer** & $115(9.4)$ \\
\hline Depression & $449(36.8)$ \\
\hline PTSD & $131(10.7)$ \\
\hline Dementia & $21(1.7)$ \\
\hline $\mathrm{CCl}$ score 0 & $395(32.6)$ \\
\hline CCl score 1-2 & $505(41.6)$ \\
\hline $\mathrm{CCl}$ score $>2$ & $313(25.8)$ \\
\hline FRS* & $24.2(22.6)$ \\
\hline
\end{tabular}

${ }^{*}$ Median and interquartile ranges are provided for AHI and FRS. All other continuous variables are reported as mean (SD).

tESS score available for 574 or 1247 patients.

‡Regular use (see online supplementary file) among patients eligible for therapy. §Includes myocardial infarction, coronary artery bypass grafting or percutaneous intervention.

IIncludes stroke, TIA, myocardial infarction, coronary artery bypass graft, percutaneous intervention and congestive heart failure.

**Includes solid tumours, leukaemia and lymphoma.

AHI, apnoea-hypopnoea index; BMI, body mass index; CCl, Charlson comorbidity index; CPAP, continuous positive airway pressure; CVD, cardiovascular disease; ESS, Epworth sleepiness scale; FRS, Framingham risk score; OSA, obstructive sleep apnoea; PTSD, post-traumatic stress disorder; TIA, transient ischaemic attack.

\section{None/mild OSA}

The largest $(\mathrm{n}=533)$ cluster was labelled 'mild', based on few disordered breathing events (median AHI (IQR) of 4 (9)), high sleep efficiency, normal REM sleep and near normal nocturnal oxygen saturation (table 3$)$. The 'PLMS' cluster $(n=119)$, with
Table 2 Description of and labels for the polysomnographic clusters based on distinguishing features

\begin{tabular}{llll}
\hline Cluster (n) & Cluster label & $\begin{array}{l}\text { Median AHI* } \\
\text { (events/hour) }\end{array}$ & $\begin{array}{l}\text { Conventional } \\
\text { OSA severity* }\end{array}$ \\
\hline A (533) & Mild & 4 & None/mild \\
B (119) & PLMS & 10 & Moderate \\
C (186) & NREM and poor sleep & 19 & \\
D (168) & REM and hypoxia & 19 & Severe \\
E (75) & Hypopnoea and hypoxia & 44 & \\
F (42) & Arousal and poor sleep & 68 & \\
G (124) & Combined severe & 84 & \\
\hline
\end{tabular}

${ }^{*}$ OSA severity definitions: none/mild ( $\left.\mathrm{AHI}<15\right)$, moderate $(15 \leq \mathrm{AHI}<30)$ and severe ( $\mathrm{AHI} \geq 30$ ). AHI was not used in generating patient clusters. Median AHIs and severity categories based on median AHI for each cluster are shown for descriptive purposes only (mean AHIs were 7.5, 13.6, 24.0, 25.0, 47.6, 72.6 and 82.4 for clusters $A, B, C, D, E, F$ and $G$, respectively).

AHI,apnoea-hypopnoea index; NREM, non-rapid eye movement; OSA, obstructive sleep apnoea; PLMS, periodic limb movements of sleep; REM, rapid eye movement.

median AHI of 10 (19), was distinguished from others primarily by a markedly higher PLMS index (PLMI). Compared with the 'mild' cluster, those in 'PLMS' spent more sleep time with oxygen saturation of $<90 \%(\mathrm{~T}<90 \%)$.

Patients in the 'mild' cluster were among the youngest, with lowest comorbidity (CCI) and CPAP use (table 4). 'PLMS' cluster members were on average 8 years older than the cases in the 'mild' group and exhibited higher comorbidity, including hypertension and diabetes (table 4, online supplementary table E1).

\section{Moderate OSA}

The median AHIs for 'NREM and poor sleep' $(n=186)$ and 'REM and hypoxia' $(n=168)$ clusters were equivalent, but the polysomnographic measures differed markedly. NREM-related apnoeas, hypopnoea-related and spontaneous arousals characterised 'NREM and poor sleep' cluster (table 3, online supplementary table E6). These patients exhibited poor sleep (55\% efficiency; $41 \%$ of stage 1 with low REM sleep). Patients in the 'REM and hypoxia' cluster exhibited the highest frequency of REM-related apnoeas, lowest oxygen saturation nadir (72.8\%) and an increased hypoxic burden (median (IQR) T<90\% of 9\% (23\%), table 3).

Patients in 'NREM and poor sleep' and 'REM and hypoxia' clusters were more obese, exhibited higher CPAP or home oxygen use and had higher prevalence of hypertension and diabetes, in comparison with the 'mild' cluster (table 4, online supplementary table E7).

\section{Severe OSA}

Cluster labelled 'hypopnea and hypoxia' ( $\mathrm{n}=75$, median AHI of 44 (39)) showed predominance of hypopnoeas with desaturations and hypoxic burden ( $\mathrm{T}<90 \%$ of $14 \%(36 \%))$, and relatively preserved sleep architecture (table 3 , online supplementary table E6). Dominance of apnoeas with arousals, but without desaturations, and markedly disturbed sleep architecture distinguished the 'arousal and poor sleep' cluster $(n=42$, median AHI of 68 (30)). Its hypoxic burden ( $\mathrm{T}<90 \%$ of $0 \%$ (4\%); oxygen saturation nadir of $87.1 \%$ ) was similar to clusters with markedly lower AHIs. The 'combined severe' cluster ( $\mathrm{n}=126$, median AHI of 84 (28)) exhibited combined apnoeas (with desaturations and arousals), severe hypoxic burden, high arousal indices and high percentage of stage 1 sleep.

Patients in the 'arousal and poor sleep' cluster tended to be older, less sleepy and have lower CPAP use, compared with those 
Table 3 Polysomnographic characteristics of OSA clusters (ranked by AHI, A-G) grouped according to domain (breathing disturbance, hypoxaemia, sleep architecture disturbance and autonomic dysregulation)*

\begin{tabular}{|c|c|c|c|c|c|c|c|}
\hline Cluster/variable & $\begin{array}{l}\text { (A) } \\
\text { Mild }\end{array}$ & $\begin{array}{l}\text { (B) } \\
\text { PLMS }\end{array}$ & $\begin{array}{l}\text { (C) } \\
\text { NREM and poor sleep }\end{array}$ & $\begin{array}{l}\text { (D) } \\
\text { REM and hypoxia }\end{array}$ & $\begin{array}{l}\text { (E) } \\
\text { Hypopnoea and } \\
\text { hypoxia }\end{array}$ & $\begin{array}{l}\text { (F) } \\
\text { Arousal and poor } \\
\text { sleep }\end{array}$ & $\begin{array}{l}\text { (G) } \\
\text { Combined severe }\end{array}$ \\
\hline $\mathrm{N}$ & 533 & 119 & 186 & 168 & 75 & 42 & 124 \\
\hline \multicolumn{8}{|c|}{ Mean \pm SD or median (IQR) } \\
\hline $\mathrm{AHI}$ & $3.6(9.0)$ & $9.9(18.6)$ & 19.3(30.6) & $18.9(24.5)$ & $44.2(39.0)$ & $67.8(30.4)$ & $83.9(28.0)$ \\
\hline \multicolumn{8}{|c|}{ Breathing disturbance } \\
\hline Total apnoea index & $3.9(5.5)$ & $5.0(11.6)$ & $13.0(26.2)$ & $13.5(18.3)$ & $13.8(22.3)$ & $61.6(29.4)$ & $77.9(28.9)$ \\
\hline Total hypopnoea Index & $3.0(12.8)$ & $6.0(16)$ & $4.0(11.0)$ & $7.0(17.0)$ & $32.0(51.0)$ & $3.5(11.0)$ & $1.0(6.0)$ \\
\hline$\%$ Obstructive apnoeas & $66 \pm 37$ & $79 \pm 29$ & $82 \pm 29$ & $89 \pm 17$ & $89 \pm 24$ & $87 \pm 24$ & $80 \pm 26$ \\
\hline$\%$ Combined apnoeas & $46 \pm 33$ & $49 \pm 31$ & $58 \pm 31$ & $55 \pm 26$ & $63 \pm 26$ & $32 \pm 22$ & $81 \pm 20$ \\
\hline$\%$ Apnoeas with arousal only & $32 \pm 34$ & $32 \pm 33$ & $30 \pm 30$ & $18 \pm 22$ & $9 \pm 17$ & $65 \pm 23$ & $7 \pm 9$ \\
\hline REM: NREM apnoea ratio & $3.4 \pm 7.5$ & $2.3 \pm 5.3$ & $0.8 \pm 3.6$ & $8.1 \pm 15.7$ & $0.3 \pm 1.1$ & $0.1 \pm 0.4$ & $0.1 \pm 0.2$ \\
\hline $\begin{array}{l}\text { Apnoea D4: apnoea arousal } \\
\text { ratio }\end{array}$ & $1.4 \pm 4.1$ & $1.2 \pm 2.7$ & $0.8 \pm 1.6$ & $3.1 \pm 4.3$ & $7.2 \pm 10.8$ & $0.1 \pm 0.1$ & $4.4 \pm 8.4$ \\
\hline \multicolumn{8}{|c|}{ Hypoxaemia } \\
\hline $\mathrm{T}<90 \% \mathrm{O}_{2}$ Index & $0.00(0.02)$ & $0.02(0.10)$ & $0.01(0.10)$ & $0.09(0.23)$ & $0.14(0.36)$ & $0.00(0.04)$ & $0.20(0.41)$ \\
\hline$>4 \%$ Desaturation index & $4.1(8.8)$ & $8.7(15.1)$ & $18.5(24.8)$ & $19.8(22.0)$ & $57.1(37.5)$ & $21.0(41.1)$ & $74.2(30.1)$ \\
\hline $\begin{array}{l}\text { Lowest nocturnal } \mathrm{O}_{2} \% \\
\text { saturation }\end{array}$ & $86.1 \pm 4.2$ & $82.7 \pm 6.7$ & $84.7 \pm 5.0$ & $72.8 \pm 7.6$ & $78.8 \pm 6.8$ & $87.1 \pm 4.2$ & $77.7 \pm 7.0$ \\
\hline \multicolumn{8}{|c|}{ Sleep architecture disturbance } \\
\hline Sleep efficiency & $77.7 \pm 14.5$ & $69.8 \pm 17.5$ & $54.7 \pm 17.9$ & $79.6 \pm 13.6$ & $68.2 \pm 16.7$ & $56.4 \pm 22.8$ & $68.8 \pm 16.6$ \\
\hline Stage $1 \%$ & $15.2(8.9)$ & $21.1(12.2)$ & $40.9(20.3)$ & $16.5(10.7)$ & $23.2(15.4)$ & $40.8(23.4)$ & $40.6(25.4)$ \\
\hline Stages 3 and $4 \%$ & $1.5(8.4)$ & $0.2(4.8)$ & $0.0(0.0)$ & $0.0(6.6)$ & $0.0(0.0)$ & $0.0(0.0)$ & $0.0(0.0)$ \\
\hline REM \% & $12.0(12.7)$ & $6.4(14.0)$ & $0.0(6.4)$ & $12.3(10.6)$ & $0.0(2.7)$ & $0.0(0.0)$ & $0.0(0.0)$ \\
\hline Stage shift index & $23.8 \pm 10.2$ & $27.3 \pm 12.2$ & $47.9 \pm 23.1$ & $24.7 \pm 12.4$ & $33.1 \pm 18.2$ & $57.3 \pm 29.1$ & $45.4 \pm 28.0$ \\
\hline \multicolumn{8}{|c|}{ Autonomic dysregulation } \\
\hline Total arousal index & $25.9 \pm 12.1$ & $40.7 \pm 21.5$ & $65.1 \pm 23.6$ & $37.3 \pm 19.8$ & $60.1 \pm 25.0$ & $88.5 \pm 27.4$ & $91.7 \pm 27.3$ \\
\hline$\%$ Spontaneous arousals & $66 \pm 22$ & $43 \pm 20$ & $50 \pm 21$ & $43 \pm 23$ & $28 \pm 16$ & $19 \pm 11$ & $13 \pm 10$ \\
\hline PLMS index & $0.0(6.2)$ & 64.7 (32.3) & $0.0(7.6)$ & $0.0(4.9)$ & $0.0(0.0)$ & $0.0(0.0)$ & $0.0(0.0)$ \\
\hline
\end{tabular}

Note: Each of the variables exhibits statistically significant differences across clusters (analysis of variance, all p values $<0.001)$.

Bold cells denote variables differentiating the clusters within the same category of OSA severity (none/mild $(\mathrm{AHI}<15)$, moderate $(15 \leq \mathrm{AHI}<30)$ and severe $(\mathrm{AHI} \geq 30)$ ).

${ }^{*}$ The above variables are provided for cluster interpretation purposes and clinical familiarity. Feature values used to generate clusters $A$ through $G$ using cluster analysis are provided in online supplementary file.

$>4 \%$ desaturation index, number of $4 \%$ desaturations/total sleep time; AHI, apnoea-hypopnoea index (events/hour of total sleep time); apnoea D4, apnoea with 4\% desaturation only and no arousal; apnoea arousal, apnoea with arousal only and no desaturation; combined apnoea, apnoea with 4\% desaturation and arousal; NREM, nonrapid eye movement; REM, rapid eye movement; OSA, obstructive sleep apnoea; PLMS, periodic limb movements of sleep; $\mathrm{T}<90 \% \mathrm{O}_{2}$ index, time spent at $\mathrm{O}_{2}$ saturation $<90 \%$ / total sleep time; total hypopnoea, hypopnoea with $4 \%$ desaturation with or without arousal.

in other clusters in the severe OSA category (table 4). This cluster also had the highest percentage of Black and Hispanic patients. Although baseline comorbidity burden was similar for clusters in the 'severe OSA' category, patients in 'arousal and poor sleep' cluster had a trend towards higher prevalence of myocardial infarction, atrial fibrillation and chronic lung disease in comparison to the 'mild' cluster (online supplementary table E7).

\section{Clinical outcomes}

After an average follow-up of $4.9 \pm 1.9$ years, 271 patients experienced ACS, TIA, stroke or death from any cause (online supplementary table E8). Statistically significant differences in Kaplan-Meier curves for the primary outcome were evident according to patient cluster (log rank, p value $<0.001$, figure 1). In unadjusted analysis (table 5), cluster membership was associated with adverse cardiovascular events or death ( $p$ value 0.002 ). Compared with the 'mild' cluster, the 'PLMS' cluster exhibited a greater than twofold higher risk for the primary outcome (hazard ratio (HR) 2.36; 95\% CI 1.61 to 3.46). Among the remaining clusters, only 'REM and hypoxia' did not exhibit a statistically higher risk of primary outcome (HR 1.34, 95\% CI 0.91 to 1.99) than 'mild' cluster. Overall, regular CPAP use was associated with a $36 \%$ decreased risk of primary outcome ( $\mathrm{p}$ value 0.0015 , table 5 ) and this attenuation varied by cluster (figure 2). After adjustment with FRS for age, sex and baseline cardiovascular risk, the association with the primary outcome remained significant for 'PLMS,' 'hypopnea and hypoxia' and 'combined severe' clusters, with the HR of 1.79 , 95\% CI 0.97 to 3.29 for the 'arousal and poor sleep' group (table 6). Because majority of combined outcome events were deaths, sensitivity analyses using CCI in place of FRS, as well as the addition of home oxygen use were performed with similar results (online supplementary tables E9 and E10). Finally, a sensitivity analysis using CVD deaths versus all-cause 
Table 4 Baseline demographic, anthropometric, sleep and comorbidity characteristics for clusters grouped by conventional OSA severity designations (based on median cluster $\mathrm{AHI}$ : none/mild (Clusters $\mathrm{A}$ and $\mathrm{B}$ ), moderate (Clusters $\mathrm{C}$ and $\mathrm{D}$ ) and severe (Clusters $\mathrm{E}, \mathrm{F}$ and $\mathrm{G})$ )

\begin{tabular}{|c|c|c|c|c|c|c|c|c|}
\hline Variable/cluster & $\begin{array}{l}\text { (A) } \\
\text { Mild } \\
\end{array}$ & $\begin{array}{l}\text { (B) } \\
\text { PLMS } \\
\end{array}$ & $\begin{array}{l}\text { (C) } \\
\text { NREM and poor } \\
\text { sleep }\end{array}$ & $\begin{array}{l}\text { (D) } \\
\text { REM and } \\
\text { hypoxia }\end{array}$ & $\begin{array}{l}\text { (E) } \\
\text { Hypopnoea and } \\
\text { hypoxia } \\
\end{array}$ & $\begin{array}{l}\text { (F) } \\
\text { Arousal and } \\
\text { poor sleep }\end{array}$ & $\begin{array}{l}(\mathrm{G}) \\
\text { Combined } \\
\text { severe }\end{array}$ & $X^{2}$ or F-stat. $\mathrm{p}$ \\
\hline \multirow[t]{2}{*}{$\mathrm{N}$} & 533 & 119 & 186 & 168 & 75 & 42 & 124 & value § \\
\hline & \multicolumn{7}{|c|}{ Mean \pm SD, Median (IQR) or N (\%) } & \\
\hline Age (years) & $55.3 \pm 11.8$ & $63.7 \pm 10.5$ & $62.0 \pm 10.8$ & $57.8 \pm 10.9$ & $59.2 \pm 11.8$ & $65.7 \pm 10.9$ & $58.0 \pm 10.9$ & $<0.0001$ \\
\hline Men & 489 (91.7) & 115 (96.6) & 184 (98.9) & $162(96.4)$ & 70 (93.3) & $40(95.2)$ & $123(99.2)$ & 0.0006 \\
\hline White & $421(81.1)$ & $103(89.6)$ & $151(83.4)$ & $115(83.9)$ & $66(88.0)$ & $29(70.7)$ & $93(75.0)$ & $<0.0001$ \\
\hline Employed & $193(37.2)$ & $29(25.2)$ & $64(35.4)$ & 62 (37.1) & $20(26.7)$ & $14(34.1)$ & 43 (34.7) & $<0.0001$ \\
\hline $\mathrm{BMI}\left(\mathrm{kg} / \mathrm{m}^{2}\right)$ & $32.4(6.4)$ & $33.7(6.5)$ & $34.7(7.7)$ & $36.8(7.0)$ & $40.0(8.5)$ & $38.8(7.9)$ & $37.2(6.9)$ & $<0.0001$ \\
\hline ESS* & $10.5 \pm 5.7$ & $9.1 \pm 5.6$ & $11.0 \pm 5.7$ & $11.1 \pm 5.8$ & $12.4 \pm 5.7$ & $10.5 \pm 6.6$ & $12.1 \pm 6.0$ & 0.0610 \\
\hline AHI (\#/hour) & $3.6(9.0)$ & $9.9(18.6)$ & 19.3(30.6) & $18.9(24.5)$ & $44.2(39.0)$ & $67.8(30.4)$ & $83.9(28.0)$ & $<0.0001$ \\
\hline None/mild OSA & 446 (83.7) & $75(63.0)$ & 76 (40.9) & $68(40.5)$ & $10(13.3)$ & $0(0)$ & $1(0.8)$ & $<0.0001$ \\
\hline Moderate OSA & $64(12.0)$ & $29(24.4)$ & $47(25.3)$ & $46(27.4)$ & $12(16.0)$ & $0(0)$ & $0(0)$ & $<0.0001$ \\
\hline Severe OSA & $23(4.3)$ & $15(12.6)$ & $63(33.9)$ & $54(32.1)$ & $53(70.7)$ & $42(100)$ & $123(99.2)$ & $<0.0001$ \\
\hline CPAP uset & $128(24.6)$ & $38(33.0)$ & $64(35.4)$ & 72 (43.1) & $37(49.3)$ & $12(29.3)$ & $51(41.1)$ & $<0.0001$ \\
\hline Smoking & 185 (36.8) & 36 (31.9) & $69(39.2)$ & $50(30.5)$ & 17 (23.6) & 12 (31.6) & $58(48.3)$ & 0.0094 \\
\hline Alcohol use & $244(48.9)$ & $49(45.4)$ & $93(55.0)$ & $78(48.7)$ & $30(41.7)$ & $20(50.0)$ & $67(56.8)$ & 0.3086 \\
\hline Drug use & $29(5.9)$ & $5(4.7)$ & $7(4.3)$ & $11(7.1)$ & $3(4.3)$ & $1(2.6)$ & $11(9.4)$ & 0.5269 \\
\hline Home oxygen & $22(4.3)$ & $11(9.7)$ & $21(11.7)$ & $16(9.9)$ & $6(8.2)$ & $2(4.9)$ & $9(7.4)$ & 0.0181 \\
\hline $\mathrm{CCl}$ score 0 & $192(37)$ & $25(22)$ & $51(28)$ & 57 (35) & $21(28)$ & $8(20)$ & 41 (34) & $<0.0001$ \\
\hline CCl score 1-2 & $217(42)$ & $51(45)$ & $72(40)$ & $65(40)$ & $35(47)$ & $22(54)$ & $43(42)$ & $<0.0001$ \\
\hline $\mathrm{CCl}$ score $>2$ & $109(21)$ & $38(33)$ & $56(31)$ & $42(26)$ & $18(24)$ & $8(27)$ & 38 (31) & $<0.0001$ \\
\hline FRS $\ddagger$ & $23.2(15.5)$ & $30.9(15.9)$ & $32.1(17.8)$ & $27.8(18.1)$ & $29.5(17.3)$ & $36.4(19.0)$ & $30.3(17.3)$ & $<0.0001$ \\
\hline
\end{tabular}

OSA severity definition: none/mild ( $\mathrm{AHI}<15)$, moderate (AHI 15 to $<30$ ) and severe (AHI $\geq 30)$.

*Data available for $43 \%, 49 \%, 44 \%, 49 \%, 49 \%, 52 \%$ and $49 \%$ of cluster $A, B, C, D, E, F$ and G members, respectively. tPer cent of those with OSA and regular CPAP use ( $>4$ hours per night) of those for whom CPAP was indicated.

¥Data available for $81.4 \%, 84 \%, 84 \%, 85 \%, 87 \%, 86 \%$ and $87 \%$ of cluster A, B, C, D, E, F and G members, respectively.

$\S$ Bonferroni corrected for multiple comparisons.

AHI, apnoea-hypopnoea index (events per hour); BMI, body mass index; CCI, Charlson comorbidity index; CPAP, continuous positive airway pressure; ESS, Epworth sleepiness scale; FRS, Framingham risk score; OSA, obstructive sleep apnoea; PLMS, periodic limb movements of sleep; NREM, non-rapid eye movement; REM, rapid eye movement.

mortality as part of the composite outcome resulted in equivalent risk associations for the clusters (data not shown).

Conventional OSA severity categories, based on AHI, were not associated with the primary outcome ( $\mathrm{p}$ value of 0.49 ) in an adjusted model, regardless of the reference group specification (none $(\mathrm{AHI}<5)$ or none/mild (AHI <15), table 6).

\section{DISCUSSION}

Using readily available polysomnographic data and unsupervised analytic methods (eg, cluster analysis), we identified multiple patient phenotypes with mean AHIs within each of the conventional, AHI-based OSA severity categories. These clusters captured different phenomena, including co-occurrence with PLMS, association of respiratory events with arousals, hypoxic load and specific sleep states. To highlight the clinical relevance of physiological heterogeneity portrayed by the clusters (but not to create a prognostic tool), we evaluated their associations with incident cardiovascular events or death. We found that cluster membership independently predicted adverse CVD or death, whereas conventional AHI-based severity categories did not.

PLMS were the main distinguishing characteristic between 'mild' and 'PLMS', the two clusters with median AHI $<15$. The high prevalence of PLMS in patients with OSA (28\%-48\%), especially those with $\mathrm{AHI}<20,{ }^{27}$ supports our finding of a 'PLMS' cluster as a group with a distinct physiological disturbance in OSA. Although PLMS are common, little is known about factors associated with their origin in patients with OSA, aside from older age and increased comorbidity burden, ${ }^{27}$ also found in the 'PLMS' cluster. Evidence of the adverse impact of PLMS on the cardiovascular system is emerging. Basal autonomic regulation is shifted towards sympathetic predominance in OSA patients with PLMS, in comparison with those with OSA alone. ${ }^{28}$ PLMS are associated with daytime blood pressure elevations ${ }^{29}$ and progression to persistent atrial fibrillation. ${ }^{30}$ After controlling for baseline cardiovascular risk, we found that the 'PLMS' cluster was associated with a twofold increase of the combined outcome compared with the 'mild' cluster and that CPAP use may attenuate this risk (figure 2). In another OSA cohort, PLMI independently predicted incidence of CVD or death from any cause. ${ }^{13}$ Taken together with our findings, evidence suggests that PLMS are important for identification of patients at increased risk of CVD or death.

In the moderate OSA severity range $(15 \leq \mathrm{AHI}<30)$, the polysomnographic characteristics of the 'NREM and poor sleep' cluster suggest that a lower arousal threshold (in addition to higher ventilatory loop gain) may result in fragmented sleep with less severe oxygen desaturation. ${ }^{31}$ Although we did not measure arousal threshold directly, this cluster exhibited a nadir oxygen 


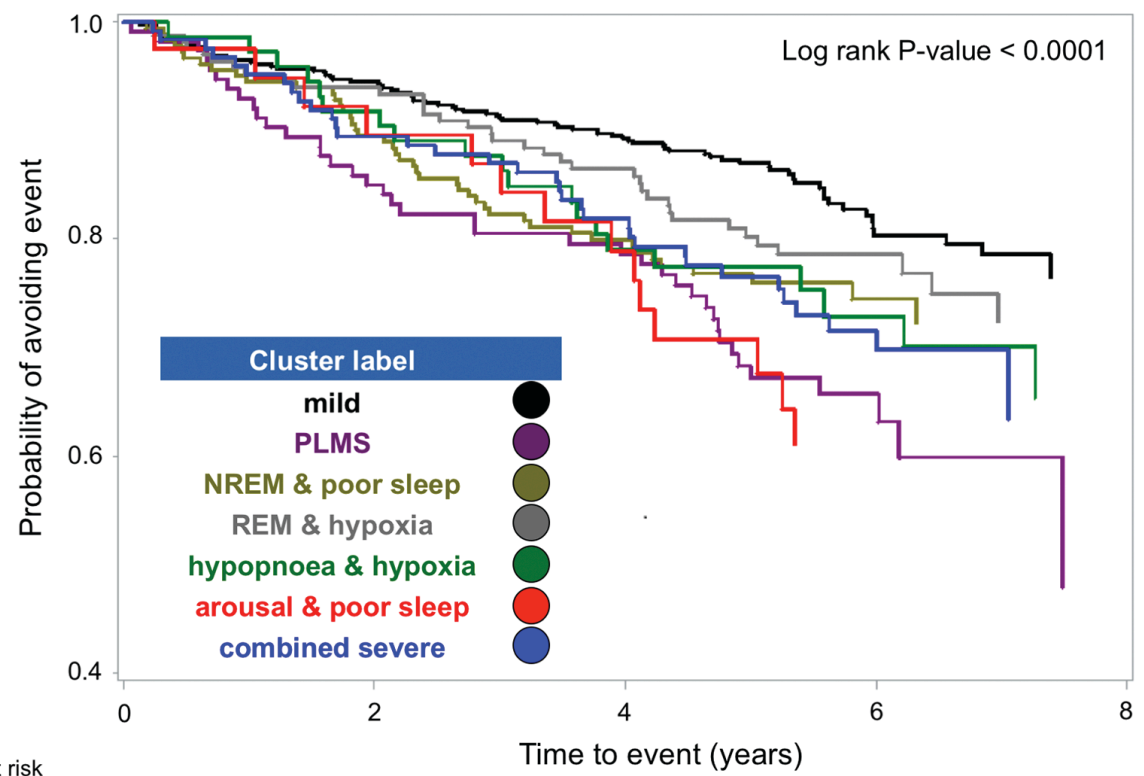

Cluster / number at risk

$\begin{array}{rc}\text { mild } & 517 \\ \text { PLMS } & 114 \\ \text { NREM \& poor sleep } & 181 \\ \text { REM \& hypoxia } & 167 \\ \text { hypopnoea \& hypoxia } & 74 \\ \text { arousal \& poor sleep } & 41 \\ \text { combined severe } & 124\end{array}$

478
96
162
154
67
34
110

Time to event (years)

$\begin{array}{ccc}407 & 129 & 17 \\ 82 & 27 & 1 \\ 130 & 42 & 5 \\ 129 & 61 & 8 \\ 51 & 26 & 5 \\ 29 & 14 & 0 \\ 95 & 40 & 3\end{array}$

Figure 1 Kaplan-Meier survival probability curves for risk of acute coronary syndrome, transient ischaemic attack, stroke or death for seven polysomnographic clusters. NREM, non-rapid eye movement; PLMS, periodic limb movements of sleep; REM, rapid eye movement.

saturation of $>82.5 \%$, a previously reported predictor of a low arousal threshold. ${ }^{32}$ In contrast, the 'REM and hypoxia' cluster, with predominance of REM-related respiratory events, severe hypoxia, but relatively non-interrupted sleep, was similar to REM-related OSA, as a previously recognised phenotype. ${ }^{233}$ In our study, the 'NREM and poor sleep' cluster was not associated with increased composite outcome, and to our knowledge, incidence of CVD or death in patients with polysomnographic characteristics similar to 'NREM and poor sleep' cluster has not been described. The 'REM and hypoxia' cluster was associated with higher (unadjusted) prevalence of hypertension (online supplementary table E7), but not with increased incidence of ACS,
TIA, stroke or death. One prior study of REM-related OSA found a lower prevalence of hypertension in comparison with non-stage-specific OSA, ${ }^{2}$ whereas another found increased prevalent and incident hypertension in OSA patients with REM AHI $>15 .{ }^{33}$ The differences in study design, outcomes and cohort characteristics (eg, predominantly male) make direct comparisons difficult.

Our findings suggest there is considerable pathophysiological variability among patients with $\mathrm{AHI} \geq 30$. Some patients may develop hypoxia at lower intensity of airway obstruction ('hypopnea and hypoxia'), compared with others who may require frank apnoeas for desaturation ('combined severe').

\begin{tabular}{|c|c|c|c|c|c|c|}
\hline \multirow{2}{*}{$\begin{array}{l}\text { Variables } \\
\text { Cluster label }\end{array}$} & \multicolumn{3}{|c|}{ Unadjusted } & \multicolumn{3}{|c|}{ Adjusted by CPAP use } \\
\hline & HR & $95 \% \mathrm{Cl}$ & $\mathrm{p}$ Value & HR & $95 \% \mathrm{Cl}$ & $\mathrm{p}$ Value \\
\hline PLMS & 2.36 & 1.61 to 3.46 & & 2.48 & 1.69 to 3.64 & \\
\hline NREM and poor sleep & 1.66 & 1.15 to 2.40 & & 1.74 & 1.20 to 2.51 & \\
\hline Arousal and poor sleep & 2.33 & 1.32 to 4.10 & & 2.42 & 1.37 to 4.26 & \\
\hline Combined severe & 1.78 & 1.19 to 2.66 & & 1.91 & 1.27 to 2.85 & \\
\hline CPAP use & & & - & & & 0.0015 \\
\hline Not regular & - & - & & Ref & & \\
\hline
\end{tabular}

\footnotetext{
*Subjects without any component of the Framingham risk score excluded.
}

AHI, apnoea-hypopnoea index (events per hour); BMI, body mass index; CCl, Charlson comorbidity index; CPAP, continuous positive airway pressure; ESS, Epworth sleepiness scale; FRS, Framingham risk score; OSA, obstructive sleep apnoea; PLMS, periodic limb movements of sleep; NREM, non-rapid eye movement; REM, rapid eye movement. 


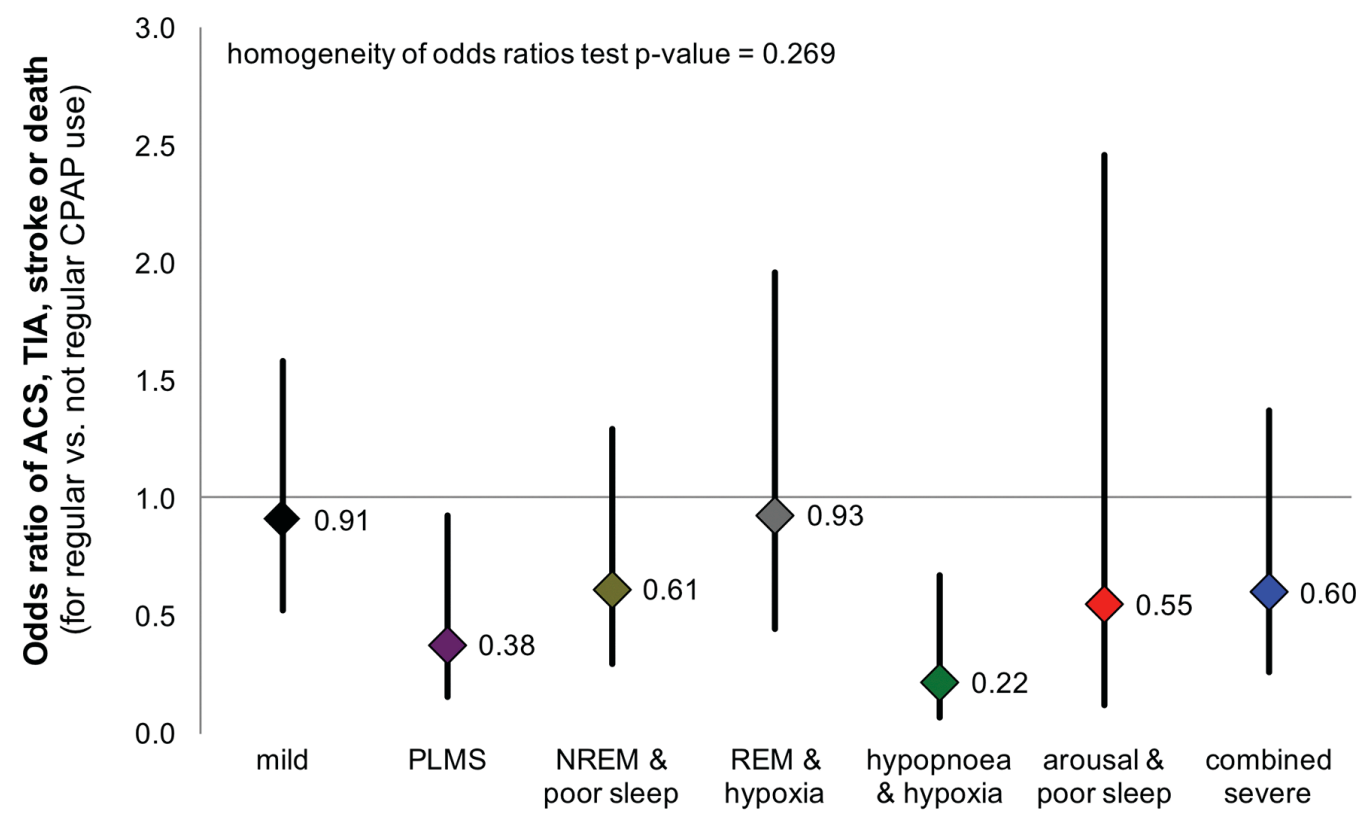

Clusters

Figure 2 Risk (odds ratio, OR) of ACS, TIA, stroke or death for those with regular versus not regular CPAP use for each cluster. ACS, acute coronary syndrome; CPAP, continuous positive airway pressure; NREM, non-rapid eye movement; PLMS, periodic limb movements of sleep; REM, rapid eye movement; TIA, transient ischaemic attack. p value is for Breslow-Day homogeneity of ORs test. OR and $95 \% \mathrm{Cl}$ reported for those with regular CPAP use versus not regular CPAP use.

Patients with equivalent AHIs and conventional arousal indices may exhibit markedly different degrees of hypoxia ('arousal and poor sleep' vs 'combined severe'). Some arousals precede airway opening, acting as a 'safety valve' to prevent worsening hypoxia/ hypercapnia, whereas others occur after the airway opening. ${ }^{31}$ In patients who open their airways prior to or without an arousal,

Table 6 Comparison of adjusted Cox proportional hazards models for primary outcome using cluster membership versus OSA severity as measured by AHI ( $n=1036^{*}$, see legend for analyses by none vs mild, moderate and severe groups)

\begin{tabular}{|c|c|c|c|c|c|c|}
\hline \multirow{2}{*}{$\begin{array}{l}\text { Variables } \\
\text { Cluster label }\end{array}$} & \multicolumn{3}{|c|}{ Cluster membership, CPAP use and Framingham risk score } & \multicolumn{3}{|c|}{ AHI severity, CPAP use and Framingham risk score } \\
\hline & HR & $95 \% \mathrm{Cl}$ & $\mathrm{p}$ Value & HR & $95 \% \mathrm{Cl}$ & $\mathrm{p}$ Value \\
\hline Mild & Ref & - & 0.0265 & - & - & \\
\hline NREM and poor sleep & 1.28 & 0.85 to 1.94 & & - & - & \\
\hline REM and hypoxia & 1.37 & 0.89 to 2.11 & & - & - & \\
\hline Combined severe & 1.69 & 1.09 to 2.62 & & - & - & \\
\hline AHI severity & & & & & & 0.5403 \\
\hline Mild/none $(\mathrm{AHI}<15)$ & - & - & & Ref & - & \\
\hline Moderate $(15 \leq \mathrm{AHI}<30)$ & - & - & & 1.21 & 0.85 to 1.73 & \\
\hline Regular & 0.62 & 0.46 to 0.84 & & 0.66 & 0.49 to 0.89 & \\
\hline $\begin{array}{l}\text { Framingham risk score } \\
\text { (change per } 10 \text { points) }\end{array}$ & 1.28 & 1.19 to 1.37 & $<0.0001$ & 1.30 & 1.22 to 1.39 & $<0.0001$ \\
\hline
\end{tabular}

Addition of ethnicity and alcohol use did not meaningfully change the cluster model results (data not shown). Significant associations with primary outcome were also not found when continuous AHI ( $p>$ value 0.2276 ) or three severity categories (none $(A H I<5$, reference) versus mild $(5 \leq A H I<15, p$ value of 0.4192$)$, moderate $(15 \leq A H I<30, p$ value of 0.1775 ) or severe (AHI $\geq 30$, $p$ value of 0.2218$)$ ) were evaluated (data not shown).

*Subjects without any component of the Framingham risk score excluded.

AHI, apnoea-hypopnoea index (events/hour of total sleep time); CPAP, continuous positive airway pressure; NREM, non-rapid eye movement; OSA, obstructive sleep apnoea; PLMS, periodic limb movements of sleep; REM, rapid eye movement. 
spontaneous arousals may potentiate ventilatory instability, interfere with progression to deeper sleep and increase cycling frequency (more frequent events with less hypoxia). ${ }^{31}$ Measuring factors such as arousal timing, apnoea and hypopnoea length, pre-event oxygen saturation, ventilatory loop gain and pharyngeal muscle responsiveness ${ }^{34}$ may help refine the differences between clusters and provide insight into these categories of risk.

The 'hypopnea and hypoxia' and 'combined severe' clusters in our study were each associated with increased risk for the composite outcome, in contrast to the AHI-based severity categories. Although many studies have reported an association between $\mathrm{AHI}$ and cardiovascular events or death, ${ }^{7-9}$ a recent systematic review shows that this association has not been found consistently. ${ }^{10}$ and, such as $\mathrm{T}<90 \%$ or a number of awakenings, have been identified as independent predictors. $^{711} 13$ It is plausible that different physiological characteristics may play an important role in some patients, but not in others. For example, for patients in the 'hypopnea and hypoxia' and 'combined severe' clusters, the duration and depth of hypoxia (not simply desaturation frequency) may pose increased risk. In contrast, for other patients ('arousal and poor sleep'), with more non-white and older individuals, the rapid cycling apnoeas associated with arousals and fragmented sleep, but without marked hypoxia, may be important. It is possible that when categorising patients using AHI alone, we may group patients with different physiological characteristics and risk profiles (online supplementary figure E3), potentially missing an opportunity for risk mitigation.

Our findings may have important implications for selecting patients for treatment and clinical trials in OSA. In current practice guidelines, for example, the recommendation for treatment of mild OSA (AHI <15) without symptoms is optional, and PLMS are not included in risk stratification. ${ }^{35}$ Focusing on AHI alone may miss a subgroup of patients at greater risk of CVD or death. CPAP use attenuated risk for the 'PLMS' cluster in our study. Limited evidence regarding CPAP treatment for unselected patients with $\mathrm{AHI}<15$ suggests no impact on fatal or non-fatal cardiovascular events. ${ }^{36}$ Our findings may also serve as a guide for recruitment into clinical trials of OSA treatment, given that patients at greatest risk of adverse outcomes may derive the greatest benefit.

Among patients traditionally classified as severe OSA, our results suggest different physiological mechanisms of sleep-disordered breathing. As such, these patients may be better served with targeted treatment based on the predominant causative physiological factors (eg, reducing arousal propensity with sedatives and/or ventilatory drive with oxygen $)^{34}$ rather than a singular approach of PAP therapy. In addition, not all patients with severe OSA may stand to benefit from treatment (figure 2). The impact of using such approaches on cardiovascular outcomes is not known and is an important area of future investigation.

Although cluster analysis has been used in OSA, ${ }^{18} 1937-40$ only two studies ${ }^{18} 19$ included polysomnographic measures other than AHI. Joosten et al ${ }^{18}$ identified six clusters in 1064 patients with mild or moderate OSA, similar to a priori defined polysomnographic OSA groups (REM-predominant, supine-isolated, supine-predominant and REM/supine overlap). Most metrics in the domains of sleep architecture, arousals and hypoxia were excluded. Analysis by Lacedonia et al, incorporating AHI, $\mathrm{T}<90 \%$ and oxygen desaturation index, identified three clusters in 198 patients with OSA. ${ }^{19}$ Two clusters with AHI $>55$ differed markedly in $\mathrm{T}<90 \%$, akin to the 'combined severe' and 'arousal and poor sleep' groups in our analysis. None of the clusters differed in prevalence of CVD or diabetes. Neither study focused on or modelled clinical outcomes by cluster, limiting the prognostic implications of the findings.

Several limitations of our study warrant consideration. Data from a single prior study were analysed (although from multiple VA medical centres), and as with most cluster analyses, our results are best used to generate hypotheses. Validation of our findings in other populations to identify distinguishing polysomnographic features is needed. Although our overall analytic sample was relatively large, because individual groups in cluster analyses may be small, we elected (a priori) not to perform split-cohort validation to avoid 'missing' potentially clinically relevant but small patient categories (eg, 'hypopnea and hypoxia', $n=75$ ). Similarly, better understanding of the cluster's implications is needed. For example, whether the 'PLMS' cluster represents restless leg syndrome with comorbid OSA, or a unique entity of OSA with PLMS, as well as its significance to cardiovascular outcomes and death, needs to be explored. Given an extensive number of polysomnographic variables included in our study $(n=29)$, and requirements of no missing data for cluster analysis, we anticipated a considerable proportion of participants from the source populations would be excluded from our analytic sample. A comparison of demographic factors, baseline cardiovascular risk comorbidities and primary outcomes between these groups revealed no differences (online supplementary table E11). In addition, our data did not include measures of symptoms, intermediate OSA phenotypes (eg, craniofacial structure and physiological traits) or cardiovascular risk biomarker data. The current study was also not designed to address the objective adherence to or effectiveness of CPAP on cardiovascular outcomes among the clusters. Nonetheless, we demonstrated increased risk of adverse events despite regular use of CPAP, suggesting that for some groups, risk was present even with treatment. Finally, the lack of gender and ethnic diversity in our cohort may limit generalisability of our findings to women or minorities.

Strengths of our study include use of a structured, datadriven approach to ascertain clinically interpretable polysomnographic features within each pathophysiological domain of OSA, and rigourous quantitative methodology (ie, cluster analysis), integrating multiple polysomnographic features to identify unique patient subgroups. Our approach is easily translatable across centres, as it is based on standard polysomnography and scoring. Assessing the clusters' incident cardiovascular outcomes or death, while adjusting for baseline cardiovascular risk using validated measures, is another strength. We also included patients with a full range of sleep apnoea severity, given that some with $\mathrm{AHI}<5$ have been previously shown to have increased risk of cardiovascular events. ${ }^{33}$ Our results serve to emphasise the need for multidimensional OSA characterisation to improve prognostication and trial design, especially as large, multidomain datasets from multiple study populations become available.

In summary, our study adds new evidence that polysomnographic heterogeneity exists within traditional OSA severity categories and that readily available data with unsupervised learning methods can be used to classify patients into subgroups reflecting different pathophysiological processes. Some of these subgroups capture the risk of adverse cardiovascular events or death otherwise missed by conventional AHI categorisation, suggesting that additional measures-such as PLMS or respiratory events with hypoxia or arousals-may be important for risk stratification and treatment selection. Future studies are needed to evaluate whether unsupervised learning methods can be applied in other populations and multidimensional datasets to better understand 
pathophysiology, improve prognostication and identify targeted treatments for patients with OSA.

\section{Author affiliations}

${ }^{1}$ Department of Medicine, Yale University School of Medicine, New Haven, Connecticut, USA

${ }^{2}$ Division of Acute Care/Health Systems, Yale School of Nursing, Yale University, New Haven, Connecticut, USA

${ }^{3}$ Department of Neurology, Yale University, New Haven, Connecticut, USA

${ }^{4}$ Departments of Neurology and Internal Medicine, Richard L. Roudenbush VA

Medical Center, Indianapolis, Indiana, USA

${ }^{5}$ Center for Outcomes Research and Evaluation, Yale University School of Medicine, New Haven, Connecticut, USA

${ }^{6}$ Section of Pulmonary, Critical Care, and Sleep Medicine, Mayo Clinic, Rochester, Minnesota, USA

${ }^{7}$ Section of Pulmonary, Critical Care, and Sleep Medicine, Case Western Reserve University, Cleveland, Ohio, USA

${ }^{8}$ Clinical Epidemiology Research Center, Veterans Affairs Connecticut Healthcare System, West Haven, Connecticut, USA

Acknowledgements The authors are grateful to US Veterans, staff at the Clinical Epidemiology Research Center at Connecticut's Veterans Affairs Medical Center and faculty and fellows of Yale's Section of Pulmonary, Critical Care and Sleep Medicine who provided valuable feedback in formulating this work.

Contributors All authors contributed substantially to the conception, design, analysis or interpretation of the data in this study. AVZ, SJ and HKY had full access to all the data in the study and take responsibility for the integrity and the accuracy of the data analyses. AVZ, SJ, BBK, NSR, JC and HKY were involved in the interpretation of data. AVZ, BBK and HKY drafted the manuscript and all authors revised it critically for important intellectual content. All authors gave final approval of this version to be submitted.

Funding This work was supported by the VA Clinical Science Research and Development (CSR\&D) Merit Review Program (CSRDS07), VA Cooperative Studies Program, NRSA Institutional training Grant from the NIH (5T32HL07778), Yale Center for Investigating Sleep Disturbance in Acute and Chronic Conditions (P20NRO14126) and Robert E. Leet and Clara Guthrie Patterson Trust Fellowship Program in Clinical Research, Bank of America, N.A., Trustee. JC is supported by the VA Cooperative Studies Program.

Competing interests None declared.

Ethics approval West Haven Veterans Affairs Institutional Review Board.

Provenance and peer review Not commissioned; externally peer reviewed.

(c) Article author(s) (or their employer(s) unless otherwise stated in the text of the article) 2018. All rights reserved. No commercial use is permitted unless otherwise expressly granted.

\section{REFERENCES}

1 Ayas NT, Owens RL, Kheirandish-Gozal L. Update in sleep medicine 2014. Am J Respir Crit Care Med 2015;192:415-20.

2 Conwell W, Patel B, Doeing D, et al. Prevalence, clinical features, and CPAP adherence in REM-related sleep-disordered breathing: a cross-sectional analysis of a large clinical population. Sleep Breath 2012;16:519-26.

3 Yamauchi M, Fujita Y, Kumamoto M, et al. Nonrapid eye movement-predominant obstructive sleep apnea: detection and mechanism. J Clin Sleep Med 2015:11:987-93.

4 Joosten SA, O'Driscoll DM, Berger PJ, et al. Supine position related obstructive sleep apnea in adults: pathogenesis and treatment. Sleep Med Rev 2014;18:7-17.

5 Palma JA, Iriarte J, Fernandez S, et al. Characterizing the phenotypes of obstructive sleep apnea: clinical, sleep, and autonomic features of obstructive sleep apnea with and without hypoxia. Clin Neurophysiol 2014;125:1783-91.

6 Burgel PR, Paillasseur JL, Roche N. Identification of clinical phenotypes using cluster analyses in COPD patients with multiple comorbidities. Biomed Res Int 2014;2014:1-9.

7 Punjabi NM, Caffo BS, Goodwin JL, et al. Sleep-disordered breathing and mortality: a prospective cohort study. PLoS Med 2009;6:e1000132.

8 Shah NA, Yaggi HK, Concato J, et al. Obstructive sleep apnea as a risk factor for coronary events or cardiovascular death. Sleep Breath 2010;14:131-6.

9 Yaggi HK, Concato J, Kernan WN, et al. Obstructive sleep apnea as a risk factor for stroke and death. N Engl I Med 2005;353:2034-41.

10 Kendzerska T, Mollayeva T, Gershon AS, et al. Untreated obstructive sleep apnea and the risk for serious long-term adverse outcomes: a systematic review. Sleep Med Rev 2014;18:49-59.
11 Stone KL, Blackwell TL, Ancoli-lsrael S, et al. Sleep disordered breathing and risk of stroke in older community-dwelling Men. Sleep 2016;39:531-40.

12 Lutsey PL, McClelland RL, Duprez D, et al. Objectively measured sleep characteristics and prevalence of coronary artery calcification: the Multi-Ethnic Study of Atherosclerosis Sleep study. Thorax 2015;70:880-7.

13 Kendzerska T, Gershon AS, Hawker G, et al. Obstructive sleep apnea and risk of cardiovascular events and all-cause mortality: a decade-long historical cohort study PLoS Med 2014;11:e1001599.

14 Weatherall M, Shirtcliffe P, Travers J, et al. Use of cluster analysis to define COPD phenotypes. Eur Respir I 2010;36:472-4.

15 Ahmad T, Pencina MJ, Schulte PJ, et al. Clinical implications of chronic heart failure phenotypes defined by cluster analysis. J Am Coll Cardiol 2014;64:1765-74.

16 Burgel PR, Paillasseur JL, Peene B, et al. Two distinct chronic obstructive pulmonary disease (COPD) phenotypes are associated with high risk of mortality. PLoS One 2012:7:e51048.

17 Jarjour NN, Erzurum SC, Bleecker ER, et al. Severe asthma: lessons learned from the National Heart, Lung, and Blood Institute Severe Asthma Research Program. Am J Respir Crit Care Med 2012;185:356-62.

18 Joosten SA, Hamza K, Sands S, et al. Phenotypes of patients with mild to moderate obstructive sleep apnoea as confirmed by cluster analysis. Respirology 2012;17:99-107.

19 Lacedonia D, Carpagnano GE, Sabato R, et al. Characterization of obstructive sleep apnea-hypopnea syndrome (OSA) population by means of cluster analysis. I Sleep Res 2016:25:724-30.

$20 \mathrm{Koo} \mathrm{BB}$, Won C, Selim BJ, et al. The Determining Risk of Vascular Events by Apnea Monitoring (DREAM) study: design, rationale, and methods. Sleep Breath 2016;20:893-900.

21 D'Agostino RB, Vasan RS, Pencina MJ, et al. General cardiovascular risk profile for use in primary care: the Framingham Heart Study. Circulation 2008;117:743-53.

22 D'Hoore W, Sicotte C, Tilquin C. Risk adjustment in outcome assessment: the Charlson comorbidity index. Methods Inf Med 1993;32:382-7.

23 Leung RS, Comondore VR, Ryan CM, et al. Mechanisms of sleep-disordered breathing: causes and consequences. Pflugers Arch 2012;463:213-30.

24 Black MH, Watanabe RM. A principal components-based clustering method to identify variants associated with complex traits. Hum Hered 2011:71:50-8.

25 Tarca AL, Carey VJ, Chen XW, et al. Machine learning and its applications to biology. PloS Comput Biol 2007:3:e116.

26 Hennig C. Cluster-wise assessment of cluster stability. Comput Stat Data Anal 2007:52:258-71.

27 Al-Alawi A, Mulgrew A, Tench E, et al. Prevalence, risk factors and impact on daytime sleepiness and hypertension of periodic leg movements with arousals in patients with obstructive sleep apnea. J Clin Sleep Med 2006;2:281-7.

28 Wu MN, Lai CL, Liu CK, et al. Basal sympathetic predominance in periodic limb movements in sleep with obstructive sleep apnea. J Sleep Res 2015;24:722-9.

29 Dean DA, Wang R, Jacobs DR, et al. A systematic assessment of the association of polysomnographic indices with blood pressure: the Multi-Ethnic Study of Atherosclerosis (MESA). Sleep 2015;38:587-96.

30 Mirza M, Shen WK, Sofi A, et al. Frequent periodic leg movement during sleep is an unrecognized risk factor for progression of atrial fibrillation. PLoS One 2013:8:e78359.

31 Eckert DJ, Younes MK. Arousal from sleep: implications for obstructive sleep apnea pathogenesis and treatment. J App/ Physiol 2014;116:302-13.

32 Edwards BA, Eckert DJ, McSharry DG, et al. Clinical predictors of the respiratory arousal threshold in patients with obstructive sleep apnea. Am J Respir Crit Care Med 2014:190:1293-300.

33 Mokhlesi B, Finn LA, Hagen EW, et al. Obstructive sleep apnea during REM sleep and hypertension. results of the Wisconsin Sleep Cohort. Am J Respir Crit Care Med 2014;190:1158-67.

34 Owens RL, Edwards BA, Eckert DJ, et al. An integrative model of physiological traits can be used to predict obstructive sleep apnea and response to non positive airway pressure therapy. Sleep 2015;38:961-70.

35 Epstein LJ, Kristo D, Strollo PJ, et al. Clinical guideline for the evaluation, management and long-term care of obstructive sleep apnea in adults. J Clin Sleep Med 2009:5:263-76.

36 Chowdhuri S, Quan SF, Almeida F, et al. An official American thoracic society research statement: impact of mild obstructive sleep apnea in adults. Am J Respir Crit Care Med 2016;193:e37-54.

37 Bailly S, Destors M, Grillet Y, et al. Obstructive sleep apnea: a cluster analysis at time of diagnosis. PLoS One 2016;11:e0157318.

38 Gagnadoux F, Le Vaillant M, Paris A, et al. RElationship between OSA clinical phenotypes and CPAP treatment outcomes. Chest 2016;149:288-90.

39 Vavougios GD, George D G, Pastaka C, et al. Phenotypes of comorbidity in OSAS patients: combining categorical principal component analysis with cluster analysis. J Sleep Res 2016:25:31-8.

40 Ye L, Pien GW, Ratcliffe SJ, et al. The different clinical faces of obstructive sleep apnoea: a cluster analysis. Eur Respir J 2014;44:1600-7. 